\title{
ANÁLISE DE BALANCEAMENTO DE CARTEIRAS DE AÇÕES DA BOVESPA ATRAVÉS DO ESPECTRO DE GRAFOS COM SINAL
}

\author{
Rafael Esteves Mansano (UFF) remansano@gmail.com \\ Renata Raposo Del-Vecchio (UFF) rrdelvecchio@gmail.com
}

\section{Resumo}

Balanceamento de carteiras de investimento é um dos fatores importantes para a análise e construção de novas carteiras. Neste estudo realizamos a análise do balanceamento de duas carteiras de investimentos baseadas nos índices Ibovespa e IDIV com dados referentes aos anos de 2017, 2018 e 2019. O método utilizado consiste em modelar as carteiras utilizando grafos com sinal e, através dos espectros das matrizes de adjacência, verificar se essas carteiras são ou não balanceadas. Além disso, é também proposto um parâmetro para a identificar em qual período as carteiras encontram-se mais desbalanceadas.

Palavras-Chaves: Bovespa; Carteira de Ações; Grafo; Grafo Balanceado

\section{Introdução}

A escolha das ações que irão compor uma carteira de investimento é uma atividade que pode levar em conta diversos critérios como, por exemplo, o perfil do investidor, o histórico de valores e até o noticiário recente. Mas, independentemente do critério utilizado, é de interesse do investidor que a carteira seja balanceada, isto é, que as ações escolhidas não precisem ser trocadas por outras com grande frequência.

Essa troca de ações, também conhecida como turnover, resulta em gastos com taxas e movimentos de compra e venda muitas vezes inoportunos, reduzindo assim a lucratividade da carteira criada. Buscando minimizar a quantidade de turnover necessário para balancear uma carteira de ações, pesquisadores como Vasanthi (2015) e Harary (2002) estão recorrendo a uma análise que utiliza grafos com sinais.

Grafos com sinais são grafos que possuem arestas que assumem apenas dois valores possíveis: “+” e “_“, sendo o sinal positivo utilizado para representar a "afinidade" entre dois vértices enquanto que o sinal negativo representa um "antagonismo". Essa representação é de grande utilidade para modelar, por exemplo, relacionamentos políticos e, o tema deste estudo, correlações de ações. 
Um grafo com sinal é dito balanceado se, e somente se, não são encontrados ciclos com um número ímpar de arestas negativas, conforme definido por Harary (1953). Partindo desse princípio, Vasanthi (2015), realizou um estudo para análise de balanceamento de carteiras de ações buscando identificar ciclos negativos em um grafo com sinal.

Dando prosseguimento à linha de pesquisa de Vasanthi (2015), este estudo fará uma análise de balanceamento de carteiras de ações também utilizando grafos com sinal. Serão utilizados dados da bolsa de valores de São Paulo. Entretanto, a análise do balanceamento será feita através do espectro destes grafos. Comparando-se os autovalores do grafo com sinal com os autovalores do grafo com todas as arestas positivas, será possível verificar se este é balanceado e ainda estabelecer uma comparação, verificando em quais anos as carteiras estiveram mais desbalanceadas.

\section{Fundamentação Teórica}

Grafos com sinal foram propostos por Harary (1953) como grafos não direcionados em que suas arestas possuem apenas dois valores possíveis: “+” e "“_. Considerando um grafo G, uma assinatura $\sigma$ é uma função de E em $\{-1,+1\}$, que atribui a cada aresta de G o valor "-1" ou “+1”, onde E é o conjunto de arestas de G. O grafo com sinal correspondente é denotado por grafos $(G, \sigma)$. Como dito anteriormente, o sinal positivo em uma aresta representa uma relação de "afinidade" entre dois vértices, enquanto que o sinal “_" representa uma relação de "antagonismo" entre dois vértices.

Além disso, um grafo com sinal é dito balanceado se nele não houver ciclo com quantidade ímpar de arestas negativas. Dessa forma, um grafo com sinal e todo positivo, isto é, sem arestas negativas, é um grafo balanceado. Este grafo é denotado por $(G,+)$, Na Figura 1 estão representados exemplos de grafos balanceados e grafos não balanceados.

Figura 1 - Exemplos de grafos balanceados e não balanceados

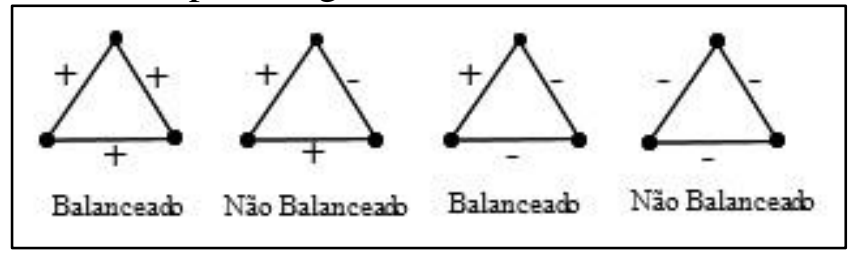


Uma operação de switch aplicada a um ou mais vértices de um grafo com sinal consiste em inverter os valores de todas as arestas ligadas a esse(s) vértice(s), conforme Figura 2. Dois grafos são considerados switching equivalents se um deles pode ser obtido do outro após uma ou mais operações de switch. Essas operações de switch possuem duas propriedades importantes para este trabalho.

Figura 2 - Operação de switch aplicada ao vértice A

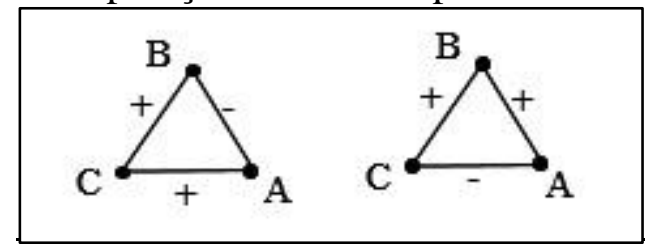

A primeira propriedade é a de que uma operação de switch não altera o balanceamento de um grafo. Portanto, um grafo não balanceado será sempre não balanceado, independentemente da quantidade de operações de switch que sejam realizadas, enquanto que um grafo balanceado permanecerá balanceado após uma ou mais operações de switch.

Assim, um grafo balanceado, ainda que possua arestas negativas, poderá, após uma sucessão de operações switch, ser transformado num grafo isomorfo, com todas as suas arestas positivas, em outras palavras, um grafo balanceado $(G, \sigma)$ e $(G,+)$ são switching equivalents. Vale ressaltar que, verificar a possibilidade de transformar um grafo com sinal em seu equivalente positivo através de sucessivas operações de switch, pode ter um grande custo computacional.

Uma das formas de representar um grafo com sinal $(G, \sigma)$ é através de sua matriz de adjacência, $A^{\sigma}=\left[a_{i j}^{\sigma}\right]$, onde $a_{i j}^{\sigma}=1$ se os vértices i e j estão ligados por uma aresta com sinal positivo, $a_{i j}^{\sigma}=-1$ se os vértices i e j estão ligados por uma aresta com sinal negativo e $a_{i j}^{\sigma}=0$ se não existe aresta ligando o vértice $\mathrm{i}$ ao vértice $\mathrm{j}$.

Dois grafos switching equivalents têm matrizes de adjacência semelhantes e, portanto, têm o mesmo espectro (mesmos autovalores). $\operatorname{Logo}$, se $(G, \sigma)$ é balanceado então $(G, \sigma)$ e $(G,+)$ são coespectrais.

Acharya (1980) provou a recíproca deste resultado, assim temos que vale o seguinte: $(G, \sigma)$ é balanceado se e somente se $(G, \sigma)$ e $(G,+)$ são coespectrais. 
Dessa forma, no caso da modelagem de uma carteira de ações através de um grafo com sinal, em que os vértices representam as ações e as arestas dependem de suas correlações, afirmar que o grafo é balanceado é afirmar que a carteira, em si, também é balanceada. Tal avaliação de balanceamento pode então ser feita através da análise espectral de dois grafos: o original e seu equivalente, com todas as arestas positivas.

\section{Metodologia}

Para este estudo foram coletados os dados de todas as ações negociadas na bolsa de valores de São Paulo (Bovespa) nos anos de 2017, 2018 e 2019. Os dados foram obtidos através do próprio site da bolsa em formato de arquivo texto posicionais agrupados por anos, meses ou dias. Os arquivos em questão são compostos por diversos dados de cada uma das ações negociadas ao longo do período selecionado, mas, para este estudo, apenas o valor das ações no fechamento diário do pregão está sendo considerado.

Para a análise desses valores e consequente geração da matriz que representa o grafo com sinal foi utilizado o aplicativo Microsoft Excel colocando, nas linhas da primeira aba da planilha, as ações negociadas no ano em questão e, nas colunas, os dias do ano em que houveram negociações.

Como exemplos de carteiras de ações, foram utilizadas as ações que compõem o índice Ibovespa, o principal índice da bolsa com 68 ações, e o IDIV (Índice Dividendos), composto pelas 34 ações que mais se destacaram em termos de remuneração na forma de dividendos. A composição desses índices é volátil, dessa forma, a configuração escolhida para esse estudo é a que estava vigente em 11 de dezembro de 2019. Apenas essas ações foram representadas nos grafos como sendo as carteiras a serem analisadas.

Foram então calculadas as variações percentuais diárias do valor das ações das carteiras selecionada. Com as variações calculadas, foi utilizada a função correl do software Excel para encontrar as correlações existentes entre cada uma das ações da carteira. O resultado desses cálculos deu origem a uma matriz quadrada e simétrica de ordem 68 , no caso do Ibovespa, e ordem 34, no caso do IDIV, nas quais a diagonal principal é composta por zeros e os outros elementos variam de -1 à +1 .

Com essa matriz de correlações obtida já seria possível criar um grafo com sinal transformando apenas seus valores positivos em arestas positivas e seus valores negativos em arestas negativas. 
Entretanto, isso resultaria em um grafo completo que não expressaria de forma justa a diferença entre correlações fortes, cujo módulo tende a 1, e correlações fracas, cujo módulo aproxima-se de 0 .

Desta forma, foram criados Fatores de Relevância para que valores de correlação acima de um determinado limite fossem representados por " +1 " e entre 0 e este limite fossem desconsiderados enquanto que valores abaixo do de outro limite fossem representados por "-1" e entre 0 e este limite fossem desconsiderados.

Em virtude da natureza das carteiras escolhidas para análise, a matriz de correlações resultante apresenta valores positivos, em média, muito mais próximos de 1 enquanto que os valores negativos muito mais próximos de 0 .

Desta forma, foram criados dois Fatores de Relevância: um para correlações positivas e outro para correlações negativas. Os fatores criados são calculados como a média das correlações positivas e a média das correlações negativas obtidas. Essa abordagem dinâmica, em virtude das carteiras escolhidas, resulta sempre em um fator de relevância positiva maior do que o fator de relevância negativa em módulo.

Assim, são então criadas as matrizes de adjacência que representam os grafos com sinal, propriamente dito, avaliando-se as correlações e levando-se em conta os Fatores de Relevância calculados. As matrizes em questão são, assim como suas antecessoras, quadradas e simétricas, e seus elementos são “+1”, representando uma aresta positiva, ou “-1”, representando uma aresta negativa, ou " 0 ", representando a ausência de uma aresta entre os vértices que, neste caso, são as ações da carteira.

Por último, consideramos os mesmos grafos, mas tomando todas as arestas positivas para calcular seus autovalores e compará-los. Se os autovalores dos dois grafos forem iguais é possível então afirmar que o grafo com sinal não possui ciclos negativos e, consequentemente, a carteira de ações em questão está balanceada. Caso contrário, a carteira de ações estará desbalanceada, forçando assim a substituição de ações através de operações de turnover diminuindo dessa forma sua lucratividade.

Com as matrizes de adjacência obtidas, foi utilizado o programa Matlab para realizar o cálculo dos autovalores e o programa Pajek para a representação dos grafos das carteiras de ações estudadas. 
Esse processo de geração dos grafos correspondentes às carteiras compostas por ações dos índices Ibovespa e IDIV foi repetido para os anos 2017, 2018 e 2019.

\section{Resultados}

Aplicando-se o método descrito, obtivemos os seguintes Fatores de Relevância de correlações:

Tabela 1 - Fatores de Relevância para os anos 2017, 2018 e 2019

\begin{tabular}{|c|c|c|c|c|c|c|}
\hline \multirow{2}{*}{} & \multicolumn{2}{|c|}{2017} & \multicolumn{2}{c|}{2018} & \multicolumn{2}{c|}{2019} \\
\cline { 2 - 7 } & Negativo & Positivo & Negativo & Positivo & Negativo & Positivo \\
\hline Ibovespa & $-0,031$ & 0,287 & $-0,095$ & 0,303 & $-0,033$ & 0,245 \\
\hline IDIV & $-0,021$ & 0,277 & $-0,049$ & 0,255 & $-0,022$ & 0,244 \\
\hline
\end{tabular}

Conforme a tabela, no ano de 2017, por exemplo, para as ações que compõem o índice Ibovespa as correlações menores ou iguais a -0,031 foram representadas na matriz de adjacência como arestas negativas. Enquanto isso, as correlações maiores ou iguais a 0,287 foram representadas como arestas positivas. Já as correlações que se encontram dentro do intervalo de -0,031 a 0,287 foram desconsideradas por não serem relevantes o suficiente.

É possível observar que as ações que compõem esses índices apresentam, em média e módulo, valores de correlação positiva muito superiores do que os valores de correlação negativa. Isso se dá em virtude da natureza dos índices, já que a valorização das ações de uma grande empresa podem ajudar a impulsionar a valorização das ações de empresas parceiras. Da mesma forma, a desvalorização das ações de uma grande empresa pode ocasionar a desvalorização das ações de empresas parceiras.

Dentro desses índices poucos são os casos de ações que invertem suas tendências de valorização e desvalorização, isto é, que apresentam correlações negativas.

Realizando-se o cálculo dos autovalores através do software Matlab para o índice Ibovespa, nos anos de 2017 e 2019 foi verificada uma diferença entre os autovalores das matrizes de adjacência dos grafos com sinal e os autovalores das matrizes de adjacência dos grafos positivos equivalentes. Já no ano de 2018, os espectros da matriz de adjacência do grafo com sinal e da matriz de adjacência do grafo positivo equivalente são iguais. 
Assim, concluímos que uma suposta carteira criada com as ações do índice Ibovespa, de 11 de dezembro de 2019, estaria desbalanceada para os anos de 2017 e 2019 mas balanceada ao longo do ano de 2018.

A distância espectral entre um grafo com sinal $(G, \sigma)$ e seu grafo subjacente positivo $(G,+)$ é dada pelo somatório dos módulos das diferenças entre os respectivos autovalores, isto é, $\sum\left|a_{i}-b_{i}\right|$. Sendo $a_{i}$ e $b_{i}$ autovalores dos grafos $(G, \sigma)$ e $(G,+)$, ordenados do menor para o maior, onde i varia de 1 à 64, no caso dos grafos relativos ao Ibovespa, e de 1 à 34 , no caso do grafo relativo ao IDIV, pois possuem, respectivamente, 64 e 34 autovalores. Usaremos então este valor, a distância espectral, como uma medida de desbalanceamento do grafo.

Verificamos que no ano de 2019 a carteira, supostamente, está mais desbalanceada $(5,45$ pontos) do que no ano de 2017 (2,55 pontos).

Embora a distância espectral tenha sido verificada, nos casos testados, como um indicativo para a análise de qual grafo encontra-se mais desbalanceado, isto é, com a maior quantidade de ciclos negativos, não foi encontrado na literatura existente estudos que comprovem que este seria um indicativo de desbalanceamento.

Com relação à análise espectral do índice IDIV, o mesmo apresentou diferença entre os autovalores da matriz de adjacência e da matriz positiva equivalente apenas no ano de 2018 . Nos anos de 2017 e 2019 os autovalores da matriz de adjacência e da matriz positiva equivalente foram iguais.

Dessa forma, concluímos que uma suposta carteira criada com as ações do índice IDIV, de 11 de dezembro de 2019, estaria balanceada nos anos de 2017 e 2019 mas desbalanceada no ano de 2018.

Como a quantidade de autovalores no espectro das matrizes referentes aos índices Ibovespa e IDIV são diferentes, já que o índice Ibovespa é composto por 68 ações enquanto o IDIV é composto por apenas 34, não é possível avaliar o nível de desbalanceamento entre esses índices utilizando a distância espectral como uma medida.

As imagens a seguir são as representações gráficas feitas através do software Pajek para as matrizes de adjacência referentes aos índices Ibovespa e IDIV nos anos de 2017, 2018 e 2019. 
Figura 3- Ibovespa em 2017 - desbalanceado

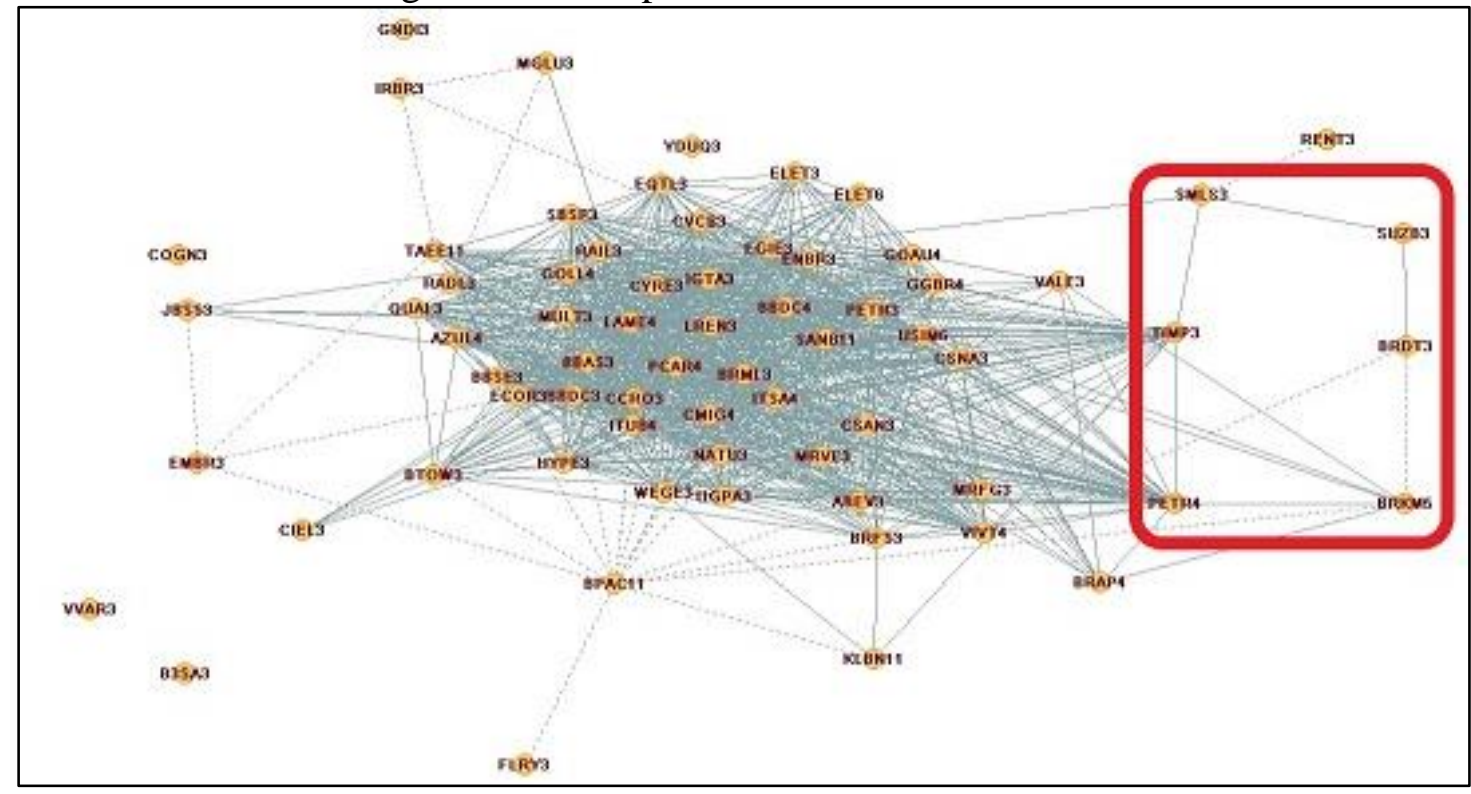

Figura 4 - Ibovespa em 2018 - balanceado

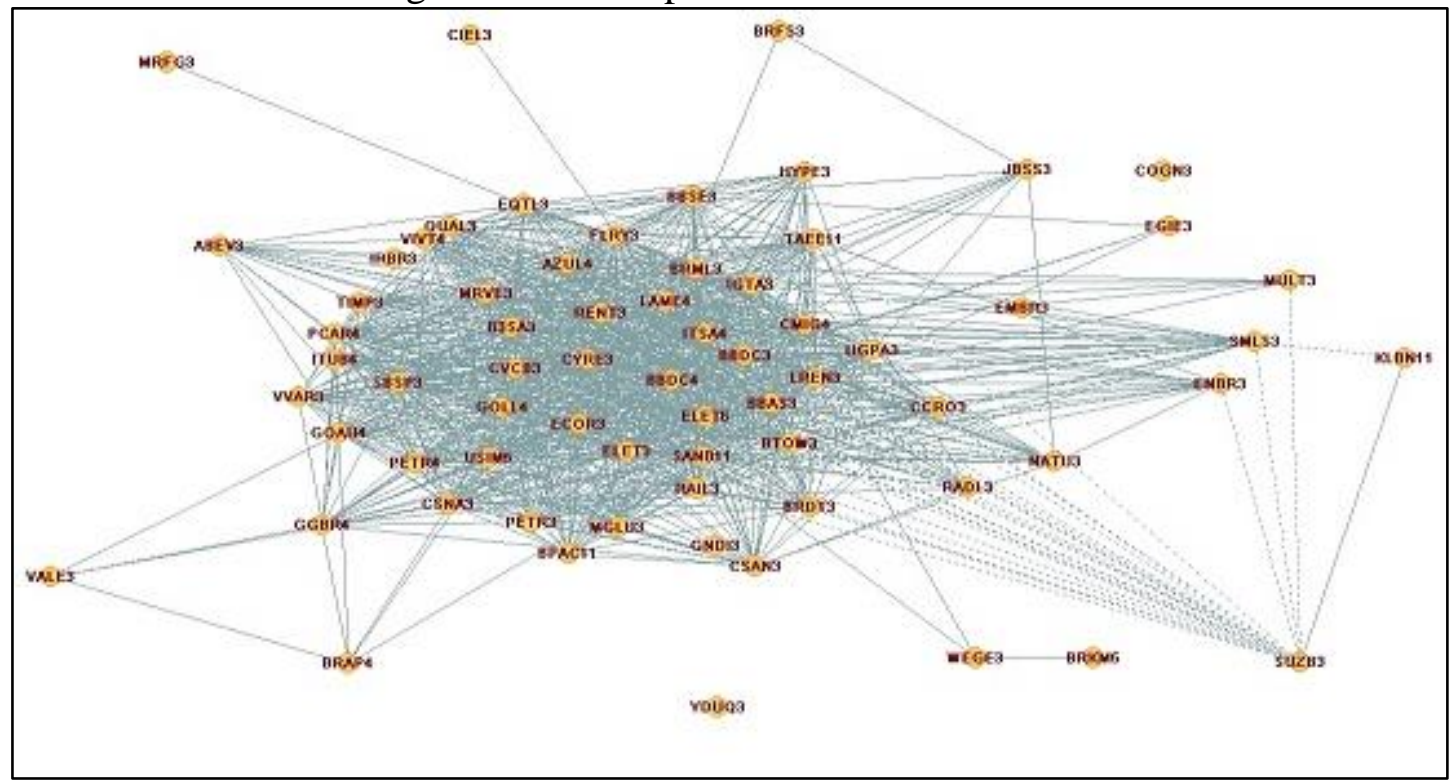


Figura 5 - Ibovespa em 2019 - desbalanceado

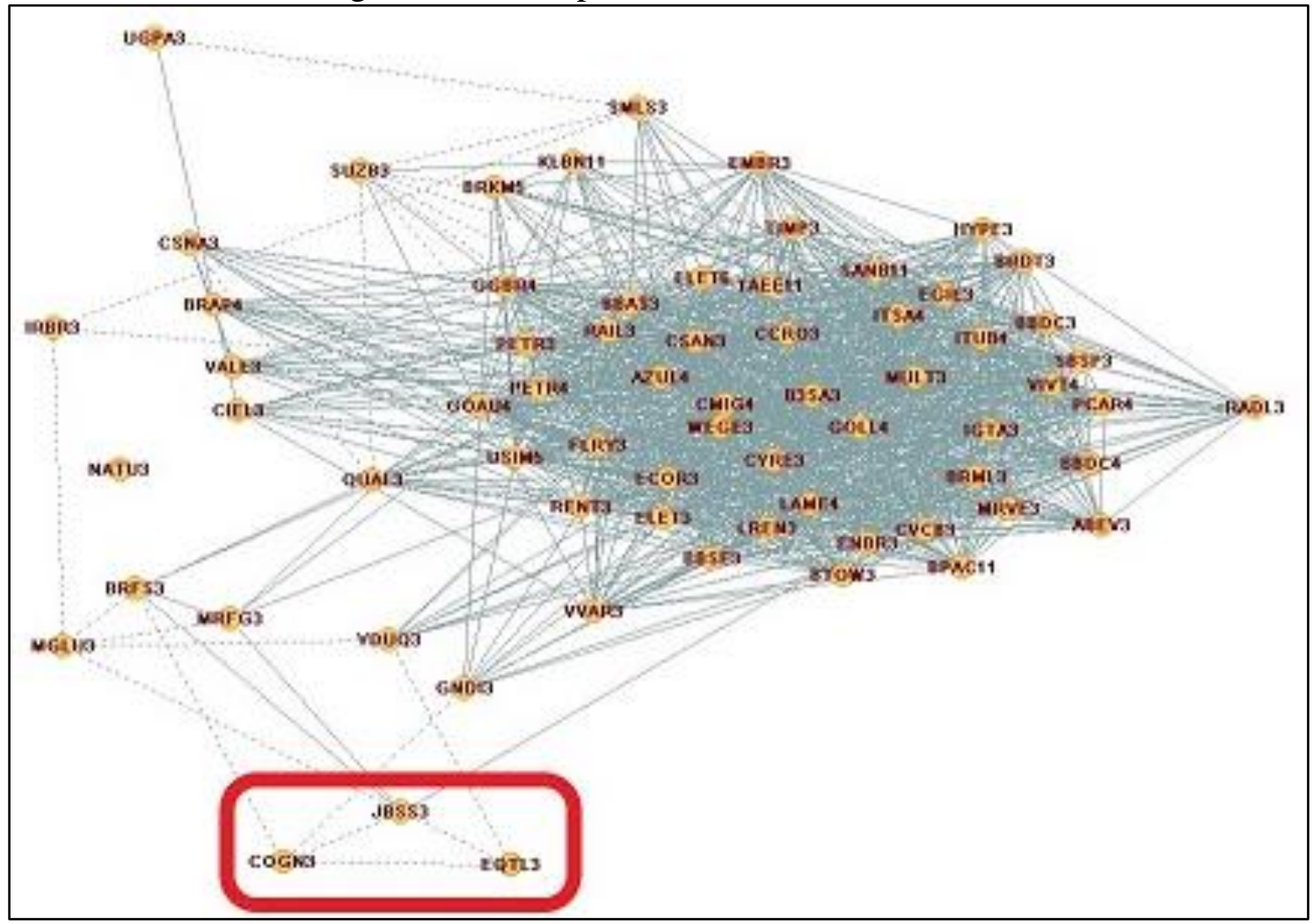

Figura 6 - IDIV em 2017 - balanceado

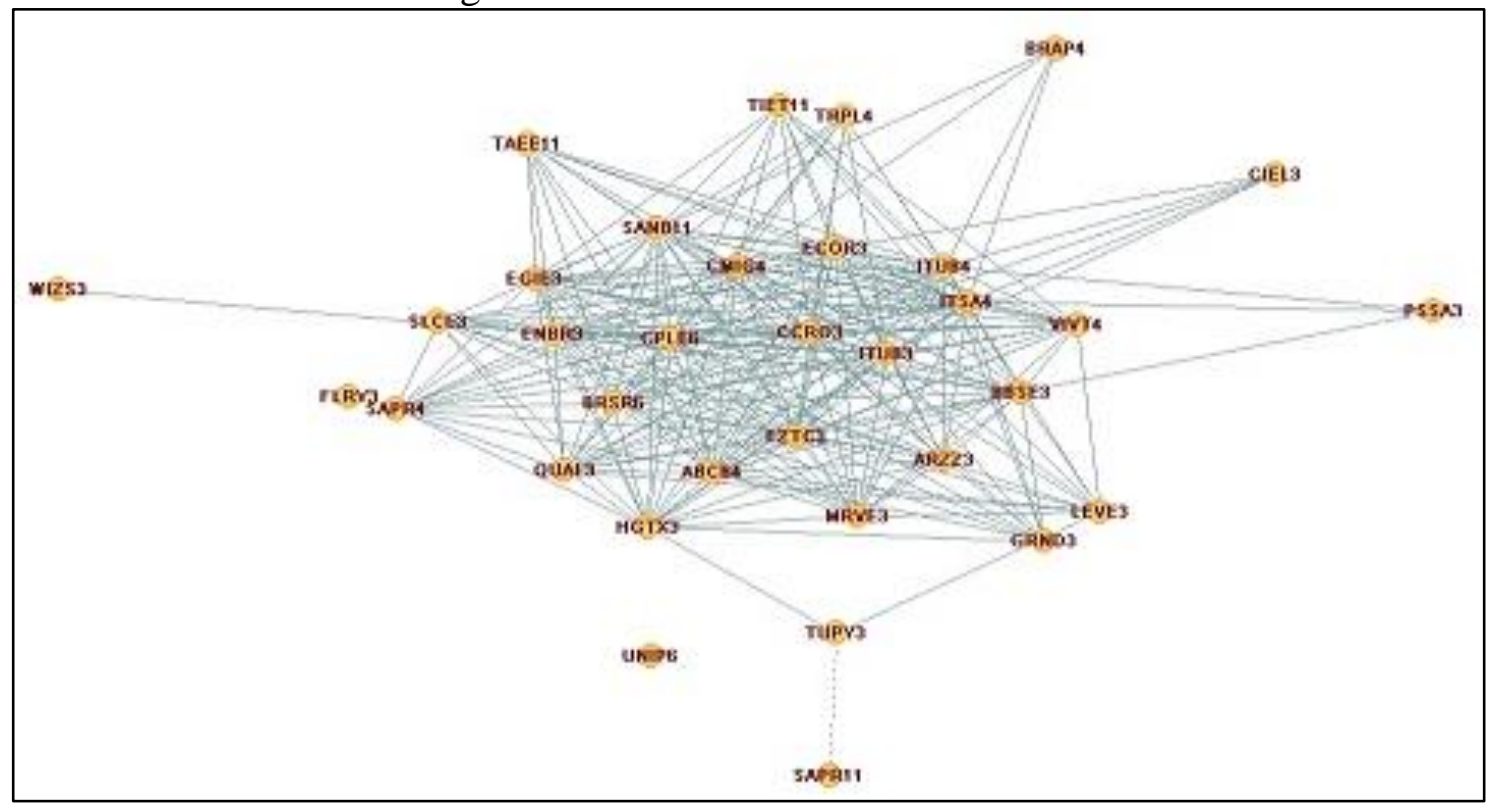


Figura 7 - IDIV em 2018 - desbalanceado

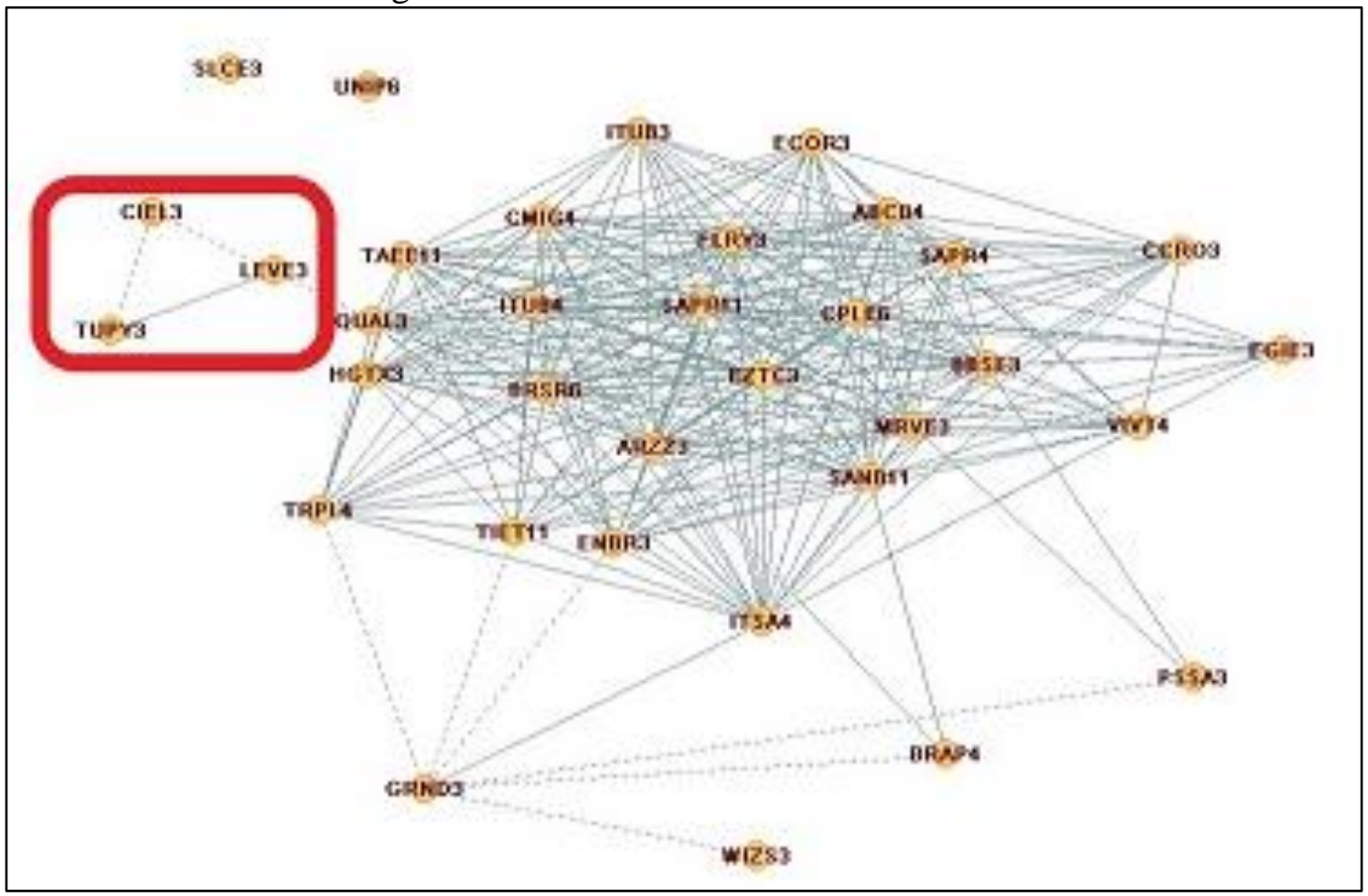

Figura 8 - IDIV em 2019 - balanceado

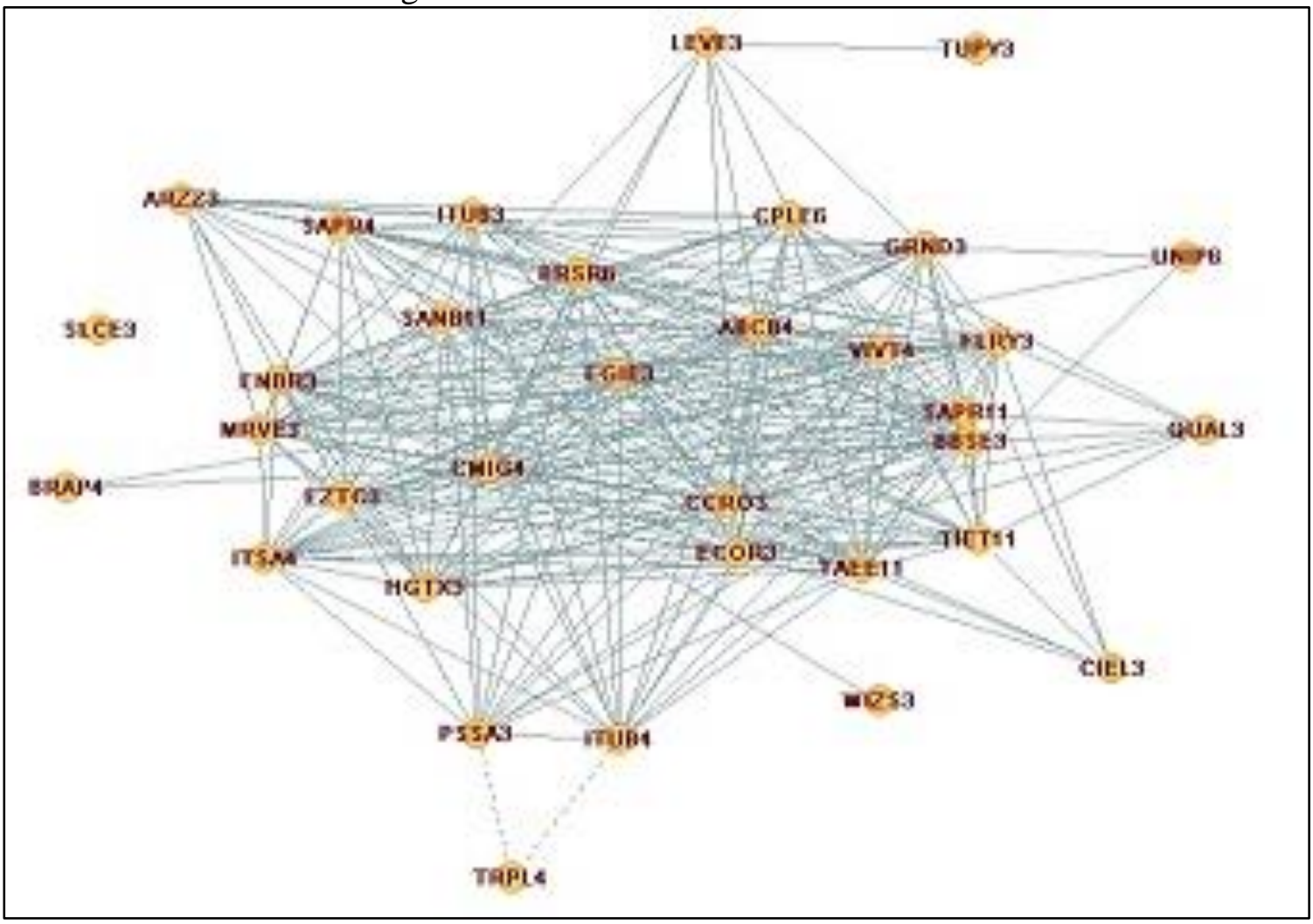


Nas figuras de 3 à 8, o nome dos vértices é o nome exato de cada uma das ações que compõem os índices na bolsa de valores. As arestas positivas da matriz de adjacência são representadas por linhas cheias e as arestas negativas representadas por linhas tracejadas. Destacado em vermelho estão alguns ciclos de valor negativo, que corroboram com os resultados obtidos através das análises espectrais dos grafos.

Entretanto, algumas considerações precisam ser feitas.

Conforme destacado por Bolt (2016), a escolha do Fator de Relevância para definir se uma correlação possui um valor suficiente para tornar-se uma aresta, positiva ou negativa, pode ser feita por diversas abordagens. Essa escolha pode significar a criação, ou não, de uma aresta que, por sua vez, pode criar, ou não, um ciclo negativo e, dessa forma, interferindo no balanceamento do grafo.

Conforme relatado anteriormente, para este estudo foi utilizado como Fator de Relevância a média do somatório das correlações e assim no ano de 2017 o índice IDIV foi apresentado com uma única aresta negativa. Essa correlação em questão (entre as ações TUPY3 e SAPR11) é de -0,066 enquanto que as outras correlações negativas deste ano estão dentro do intervalo entre 0,010 e 0. Dessa forma, apenas a correlação negativa entre as ações TUPY3 e SAPR11 foi representada no grafo já que as outras foram eliminadas através do Fator de Relevância.

Outro ponto que merece destaque é o fato de que algumas ações que compõem os índices em 11 de dezembro de 2019 não estavam sendo comercializadas nos anos anteriores. Dessa forma, tais ações, durante a aplicação do método, não possuíam qualquer tipo de correlação com outras ações e, assim, foram representadas nos grafos como vértices sem arestas.

Vale ressaltar, porém, que algumas ações também foram representadas como vértices sem arestas, apesar de possuírem correlação com outras ações, como por exemplo a ação NATU3 do índice Ibovespa no ano de 2019. Contudo, são casos em que as correlações não foram representadas por não satisfazerem os critérios criados pelos Fatores de Relevância.

Como estudo futuro sugerimos a utilização deste método para análise das correlações entre os principais índices das diversas bolsas de valores mundiais, um estudo para comprovar a eficácia da Distância Espectral como uma medida de desbalanceamento e uma análise de qual a forma mais adequada para o cálculo do Fator de Relevâncias. 


\section{Referências}

ACHARYA, B. Devadas. Special criterion for cycle balance in networks. Journal of Graph Theory, Volume 4, Issue 1.1980. Pages 1-11

HARARY, Frank. On the notion of balanced in a signed graph. Michigan Math. 1953. Pages 143-146

HARARY, Frank; LIM, M-H. and WUNSCH, D.C. Signed graphs for portfolio analysis in risk management. IMA Journal of Management Mathematics. 2002. Pages 201-210

TAYLOR, Bolt; HAMPTON, Ryan S.; FURR, R. Michael; FLEESON, William; LAURIENTI, Paul J. and DAGENBACH, Dale. Integrating Personality/Character Neuroscience with Network Analysis. 2016.

VASANTHI, B.; ARUMUGAN, S.; NAGAR, A. and MITRE, S.; Applications of signed graphs to Portfolio Tunover Analysis. Procedia - Social and Behavioral Sciences, 2015. Pages 1203 - 1209. 